\title{
A Time-Space Collocation Spectral Approximation for a Class of Time Fractional Differential Equations
}

\author{
Fenghui Huang \\ Department of Mathematics, School of Sciences, South China University of Technology, \\ Guangzhou 510641, China \\ Correspondence should be addressed to Fenghui Huang, huangfh@scut.edu.cn
}

Received 22 May 2012; Revised 26 July 2012; Accepted 29 July 2012

Academic Editor: Fawang Liu

Copyright (C 2012 Fenghui Huang. This is an open access article distributed under the Creative Commons Attribution License, which permits unrestricted use, distribution, and reproduction in any medium, provided the original work is properly cited.

\begin{abstract}
A numerical scheme is presented for a class of time fractional differential equations with Dirichlet's and Neumann's boundary conditions. The model solution is discretized in time and space with a spectral expansion of Lagrange interpolation polynomial. Numerical results demonstrate the spectral accuracy and efficiency of the collocation spectral method. The technique not only is easy to implement but also can be easily applied to multidimensional problems.
\end{abstract}

\section{Introduction}

Fractional differential equations have attracted in recent years considerable interest because of their ability to model complex phenomena. For example, fractional derivatives have been used successfully to model frequency-dependent damping behavior of many viscoelastic materials. They are also used in modeling of many chemical processes, mathematical biology, and many other problems in engineering. Related equations of importance are fractional diffusion equations, the fractional advection-diffusion equation for anomalous diffusion with sources and sinks, and the fractional Fokker-Planck equation for anomalous diffusion in an external field, and so forth.

In this paper, we consider the following time fractional differential equation (TFDE)

$$
\begin{aligned}
D_{t}^{\mu} u(x, t) & =-\lambda^{2} u(x, t)-v \frac{\partial u(x, t)}{\partial x}+D \frac{\partial^{2} u(x, t)}{\partial x^{2}}+f(x, t) \\
& =\mathcal{L} u(x, t)+f(x, t), \quad a<x<b, t>0,
\end{aligned}
$$




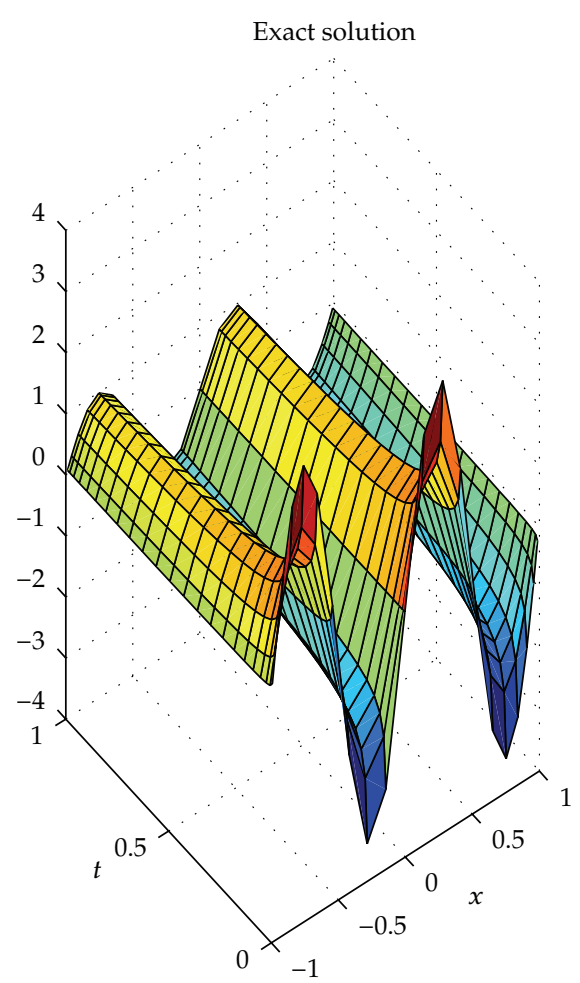

(a)



(b)

Figure 1: Exact and numerical solution with, $\mu=0.8, \beta=-0.2$.

where $\mathcal{L} u(x, t)=-\lambda^{2} u(x, t)-(v(\partial u(x, t) / \partial x))+\left(D\left(\partial^{2} u(x, t) / \partial x^{2}\right)\right)$ is a linear differential operator. $\lambda, v \geq 0, D>0$ are given constants, $0<\mu \leq 1, f(x, t)$ is a given continuous function, $D_{t}^{\mu} u(x, t)$ is a time fractional derivative which is defined in the Caputo sense

$$
D_{t}^{\alpha} f(t)= \begin{cases}\frac{\partial^{m} f(t)}{\partial t^{m}}, & \alpha=m \in \mathbb{N}, \\ \frac{1}{\Gamma(m-\alpha)} \int_{0}^{t}(t-\tau)^{m-\alpha-1} \frac{\partial^{m} f(\tau)}{d \tau^{m}} d \tau, & m-1<\alpha<m .\end{cases}
$$

The use of Caputo derivative in the above equation is partly because of the convenience to specify the initial conditions [1].

The TFDE (1.1) includes a few special cases: time fractional diffusion equation, time fractional reaction-diffusion equation, time fractional advection-diffusion equations, and their respective corresponding integer-order partial differential equations.

There are many analytical techniques for dealing with the TFDE, such as integral transformation method (including Laplace's transform, Fourier's transform, and Mellin's transform) [1-5], operational calculus method [6], Adomian decomposition method [7], iteration method and series method [8], and the method of separating variables [9].

One of the key issues with numerical solution of the TFDE (1.1) is design of efficient numerical schemes for time fractional derivative. Until now, most numerical algorithms have 


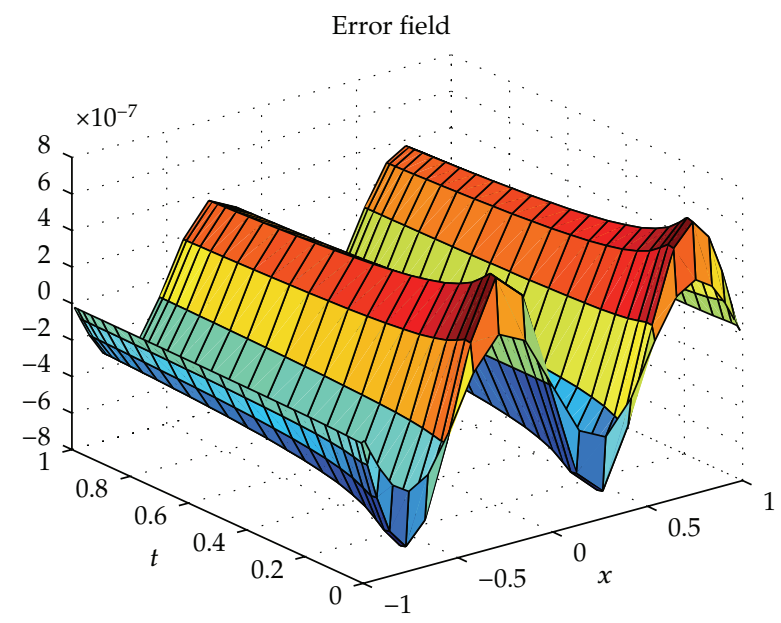

Figure 2: Error filed for $\mu=0.8, \beta=-0.2$.

relied on the finite difference (FD) methods to discretize the fractional derivatives, and the numerical accuracy always dependent on the order of the fractional derivatives. On the other hand, those FD methods have been generally limited to simple cases (low dimension or small integration) and are very difficult to improve the numerical accuracy [10-14]. Some numerical schemes using low-order finite elements (FE) have also been proposed [15-17]. The fractional derivatives are defined using integrals, so they are nonlocal operators. This nonlocal property means that the next state of a system not only depends on its current state but also on its historical states starting from the initial time. This nonlocal property is good for modeling reality, but they require a large number of operations and a large memory storage capacity when discretized with low-order FD and FE schemes. From this point, the "global method"- the nonlocal methods, like the spectral method-is well suited to discretize the nonlocal operators like fractional-order derivatives. These methods naturally take the global behavior of the solution into account and thus do not result in an extra computational cost when moving from an integer order to a fractional-order model. For example, Hanert has proposed a pseudospectral method based on Chebyshev basis functions in space and Mittag-Leffler basis functions in time to discretize the time-space fractional diffusion equation $[18,19]$. Li and $\mathrm{Xu}$ have proposed a Galerkin spectral method based on Lagrangian basis functions in space and Jacobi basis functions in time for time fractional diffusion equation [20].

In this paper, we propose a time-space collocation spectral method to discretize the TFDEs (1.1), which is easier to implement and apply to multidimensional problems than the existing Galerkin spectral. Another advantage of the present scheme is that the method can easily handle all kinds of boundary conditions.

\section{Analytical Solution of the TFDE in a Bounded Domain}

In this section, we present some analytical solutions of the TFDE which will be found helpful in the comprehension of the nature of such a problem. 


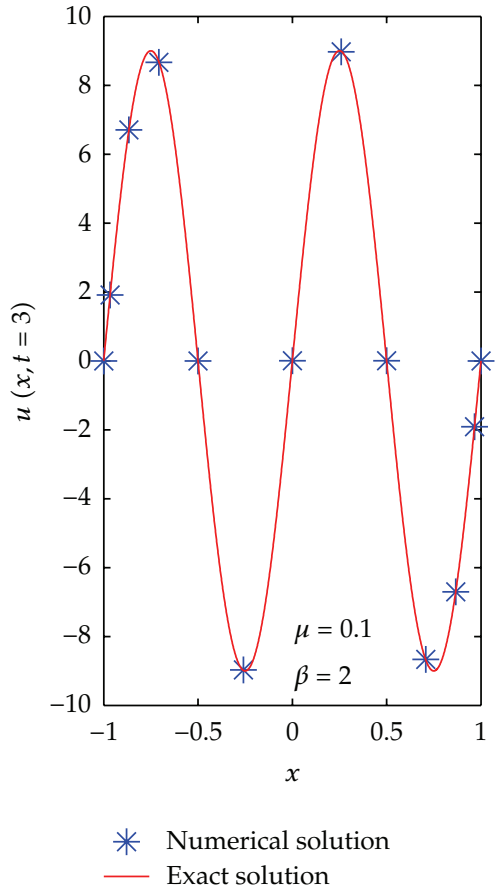

(a)

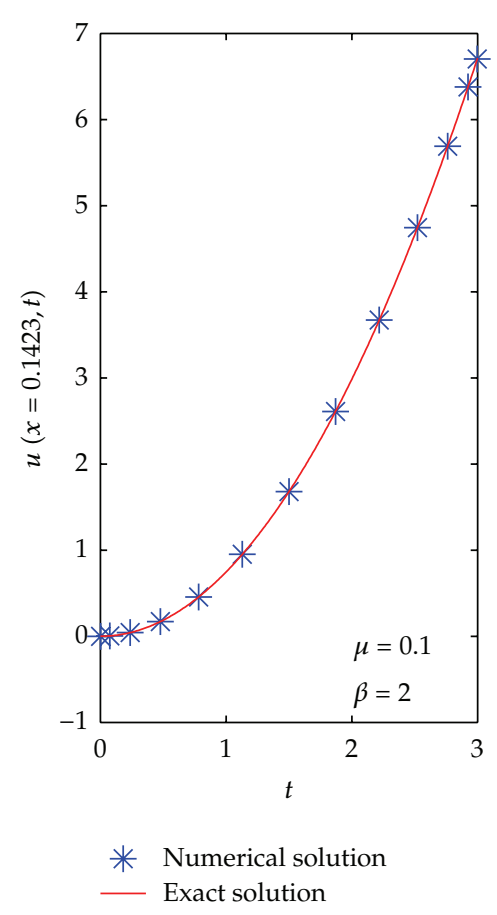

(b)

Figure 3: (a) The comparison of the exact solution and numerical solution when $t=3$. (b) The comparison of the exact solution and numerical solution when $x=0.7557$.

We consider the TFED (1.1) with initial condition

$$
u(x, 0)=\phi(x), \quad x \in(a, b)
$$

and Dirichlet boundary conditions

$$
u(a, t)=\varphi_{1}(t), \quad u(b, t)=\varphi_{2}(t), \quad t \in(0, T),
$$

or Neumann boundary conditions

$$
u_{x}(a, t)=\varphi_{1}(t), \quad u_{x}(b, t)=\varphi_{2}(t), \quad t \in(0, T)
$$




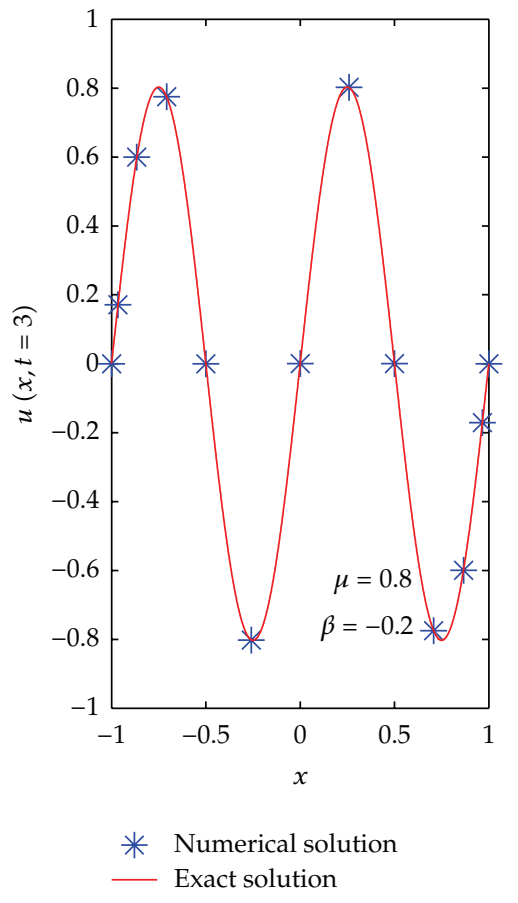

(a)

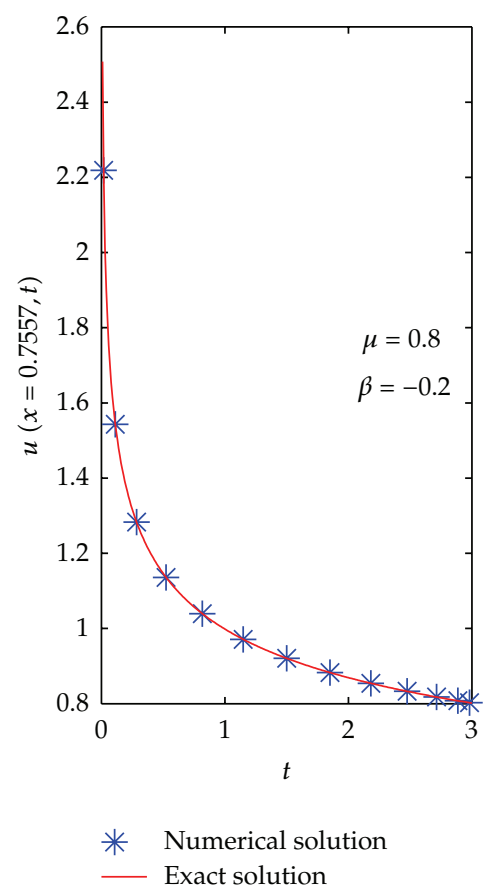

(b)

Figure 4: (a) The comparison of the exact solution and numerical solution when $t=3$. (b) The compare of the exact solution and numerical solution when $x=0.7557$.

For the case that $f \equiv 0$ and $a=0, \varphi_{1}(t)=\varphi_{2}(t)=0$, by applying the finite sine (cosine) and Laplace transforms to (1.1) with initial condition (2.1), the analytical solutions for the problem can be obtained [5] as

$$
\begin{aligned}
u(x, t)= & \frac{2 e^{v x / 2 D}}{b \sqrt{D}} \sum_{n=1}^{\infty} E_{\mu}\left(-\left[\frac{v^{2}}{4 D}+\lambda^{2}+\left(\frac{n \pi}{b}\right)^{2}\right] t^{\mu}\right) \sin \left(\frac{n \pi x}{b \sqrt{D}}\right) \\
& \times \int_{0}^{b \sqrt{D}} \phi(y) \sin \left(\frac{n \pi y}{b \sqrt{D}}\right) e^{\nu y / 2 D} d y,
\end{aligned}
$$

for homogeneous Dirichlet boundary conditions, and

$$
\begin{aligned}
u(x, t)= & \frac{2 e^{\nu x / 2 D}}{b \sqrt{D}} \sum_{n=1}^{\infty} E_{\mu}\left(-\left[\frac{v^{2}}{4 D}+\lambda^{2}+\left(\frac{n \pi}{b}\right)^{2}\right] t^{\mu}\right) \cos \left(\frac{n \pi x}{b \sqrt{D}}\right) \\
& \times \int_{0}^{b \sqrt{D}} \phi(y) \cos \left(\frac{n \pi y}{b \sqrt{D}}\right) e^{\nu y / 2 D} d y,
\end{aligned}
$$




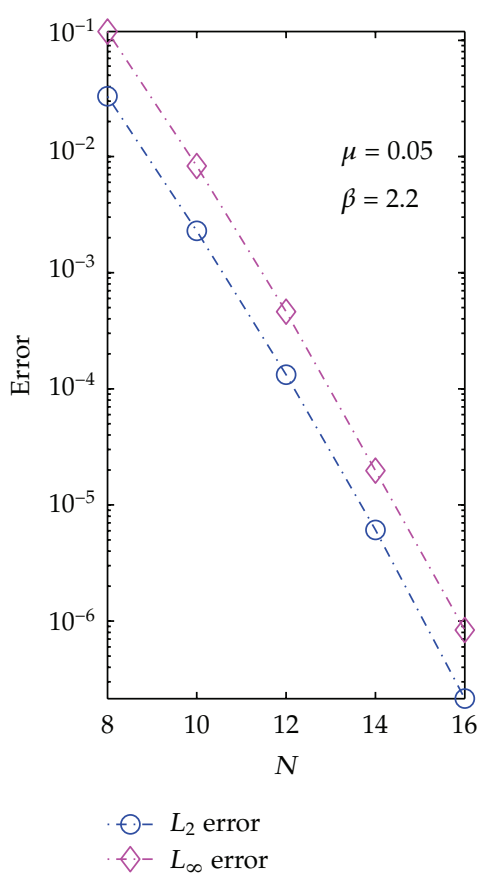

(a)

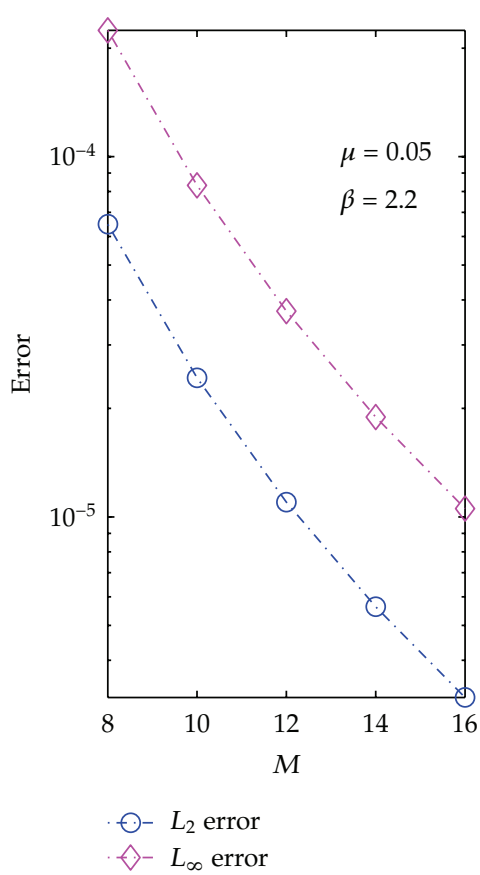

(b)

Figure 5: $y$-axis is $\log$ scale. (a) Errors versus $N$ with $M=16, \mu=0.05$. (b) Errors versus $M$ with $N=$ $16, \mu=0.05$.

for homogeneous Neumann boundary conditions. Where $E_{\alpha}(z)$ denotes a one-parameter Mittag-Leffler function which is defined by the series expansion

$$
E_{\alpha}(z):=\sum_{k=0}^{\infty} \frac{z^{k}}{\Gamma(\alpha k+1)}, \quad z \in \mathbb{C},(\alpha>0)
$$

Obviously, if we fix the variable $x=x^{*}$, that is, $u(\cdot, t)$ is a function of the variable $t$, we can see the solution $u(\cdot, t)$ is not smooth on $[0, T]$. According to $(2.4)$ and (2.5), its first derivative behaves like $u^{\prime}(\cdot, t) \sim t^{\mu-1}$ and the high-order derivative behaves like $u^{(m)}(\cdot, t) \sim$ $t^{\mu-m}$ near $t=0^{+}$.

\section{Collocation Spectral Method}

First, we give the properties of the Caputo fractional derivative [1] as

$$
J^{\beta}\left(D^{\beta} g(t)\right)=g(t)-\sum_{k=0}^{n-1} g(0) \frac{t^{k}}{k !}, \quad 0 \leq n-1<\beta<n
$$






(a)

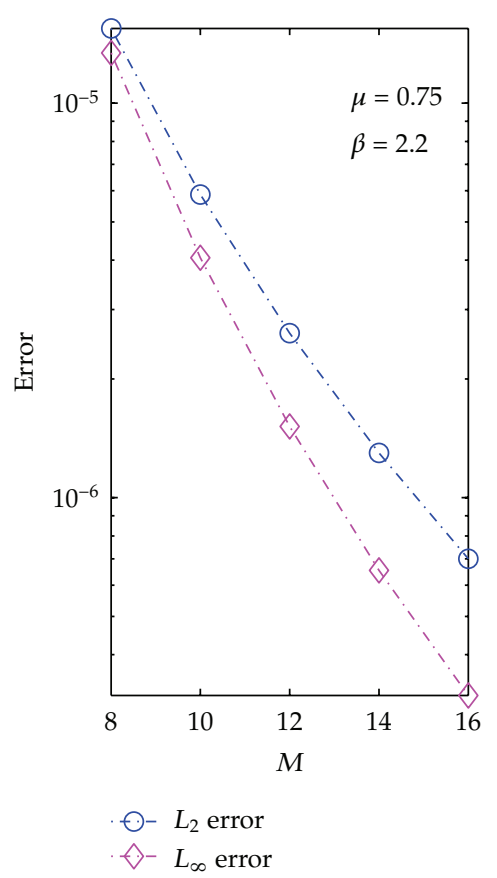

(b)

Figure 6: $y$-axis is $\log$ scale. (a) Errors versus $N$ with $M=16, \mu=0.75$. (b) Errors versus $M$ with $N=$ $16, \mu=0.75$.

where $J^{\beta}$ is the Riemann-Liouville fractional integral of order $\beta$ which is defined by

$$
J^{\beta} g(t):=\frac{1}{\Gamma(\beta)} \int_{0}^{t}(t-\tau)^{\beta-1} g(\tau) d \tau
$$

By the above properties, we can transform the initial value problem (1.1) into the following Volterra integral equation equivalently:

$$
u(x, t)-u(x, 0)=\frac{1}{\Gamma(\mu)} \int_{0}^{t} \frac{\mathcal{L} u(x, \tau)}{(t-\tau)^{1-\mu}} d \tau+\frac{1}{\Gamma(\mu)} \int_{0}^{t} \frac{f(x, \tau)}{(t-\tau)^{1-\mu}} d \tau
$$

For the singular behavior of the exact solution near $t=0^{+}$which we have mentioned in the special case (the exact solution (2.4) or (2.5) behaves $u^{\prime}(\cdot, t) \sim t^{\mu-1}$ near $t=0^{+}$), the direct application of the spectral methods is difficult. To overcome this difficulty, we use the technique in [21], that is, applying the transformation

$$
\bar{u}(x, t)=t^{1-\mu}[u(x, t)-u(x, 0)],
$$




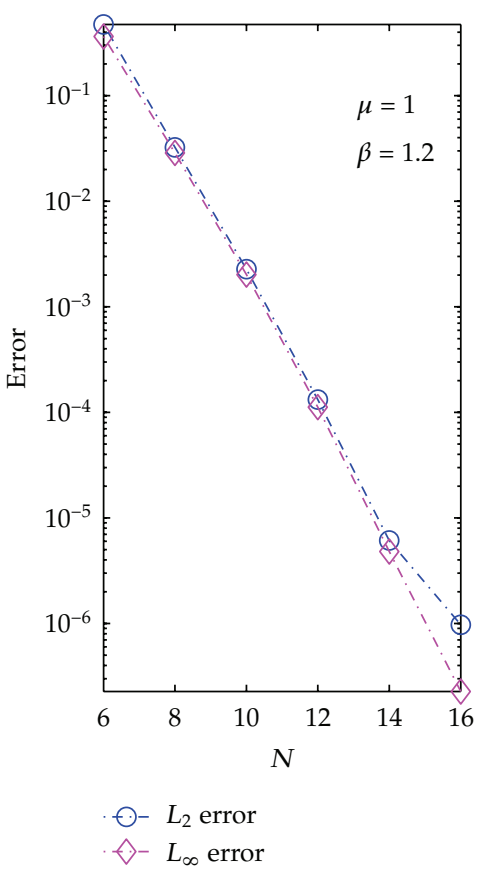

(a)

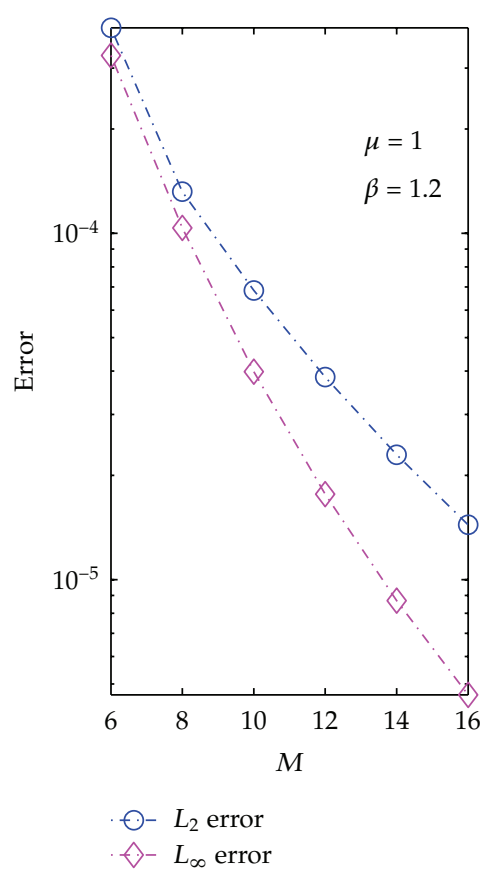

(b)

Figure 7: $y$-axis is $\log$ scale. (a) Errors versus $N$ with $M=16, \mu=1$. (b) Errors versus $M$ with $N=16, \mu=1$.

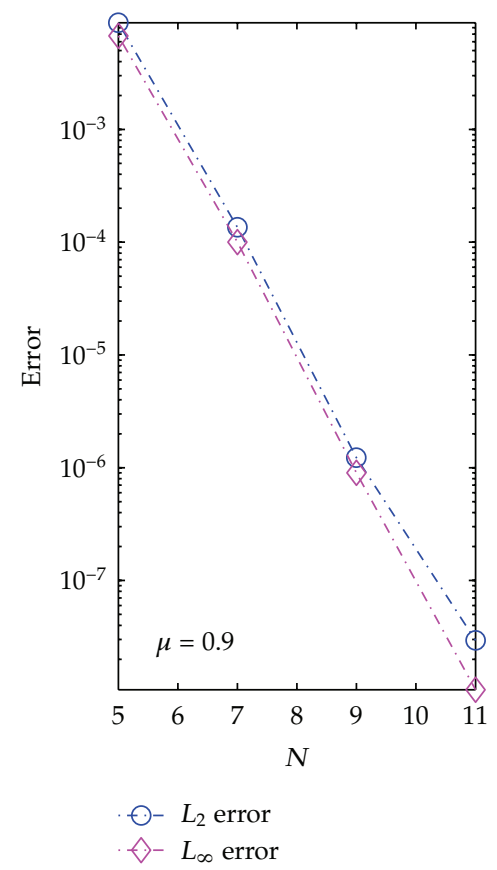

(a)

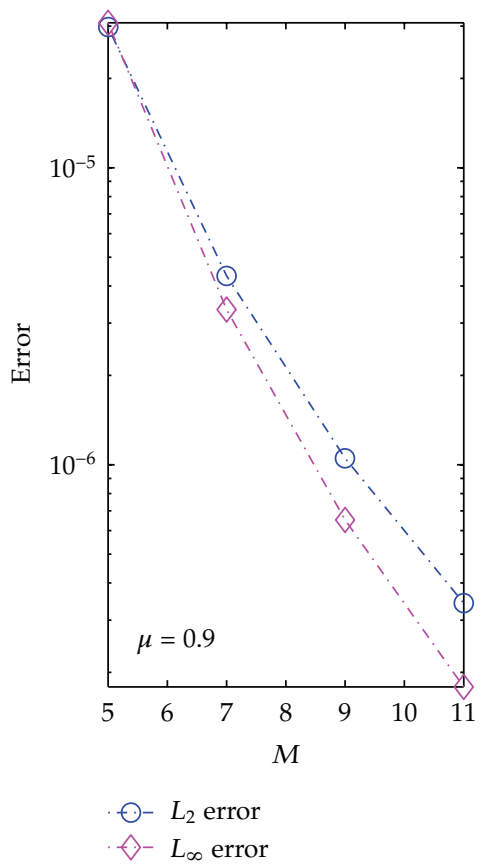

(b)

Figure 8: $y$-axis is $\log$ scale. (a) Errors versus $N$ with $M=16, \mu=0.9$. (b) Errors versus $M$ with $N=16, \mu=$ 0.9 . 


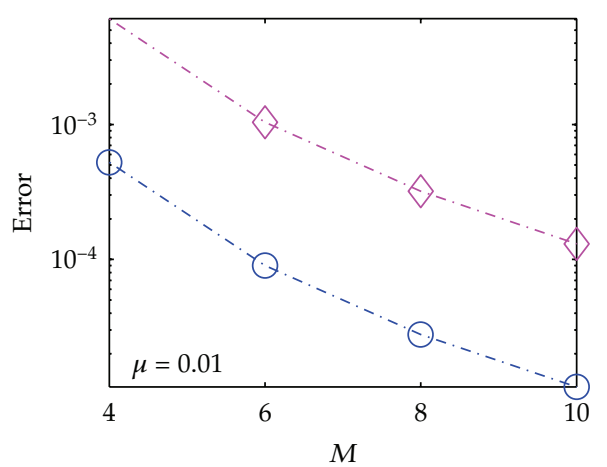

(a)

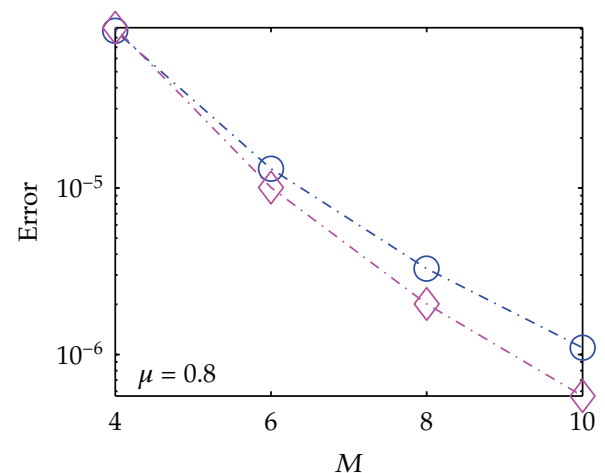

- $-L_{2}$ error

. $L_{\infty}$ error

(c)

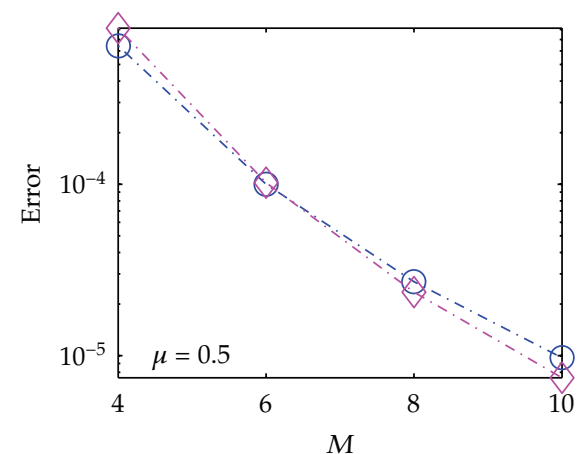

(b)

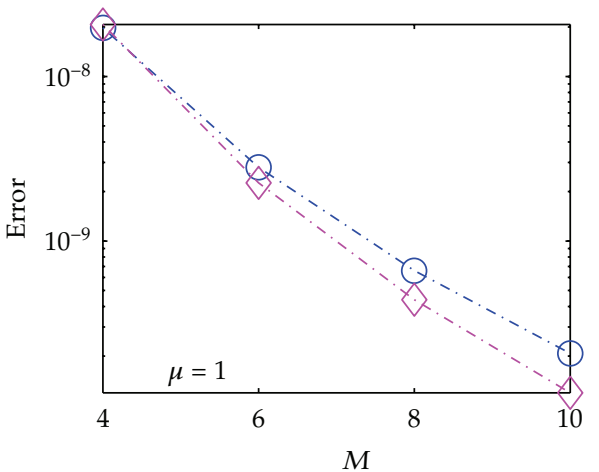

$\odot-L_{2}$ error

- $L_{\infty}$ error

(d)

Figure 9: $y$-axis is $\log$ scale. Errors versus $M$ with $N=16$ for several $\mu$.

to make the solution smooth. Then (3.3) is transformed to the equation

$$
\bar{u}(x, t)=\bar{f}(x, t)+\frac{t^{1-\mu}}{\Gamma(\mu)} \int_{0}^{t} \frac{\mathcal{L} \bar{u}(x, \tau)}{(t-\tau)^{1-\mu} \tau^{1-\mu}} d \tau,
$$

where

$$
\bar{f}(x, t)=\frac{t^{1-\mu}}{\Gamma(\mu)} \int_{0}^{t} \frac{f(x, \tau) d \tau}{(t-\tau)^{1-\mu}}+\frac{\mathcal{L} u(x, 0)}{\Gamma(1+\mu)} t .
$$

To apply the theory of orthogonal polynomials, we set

$$
t=\frac{T}{2}(1+y), \quad y \in[-1,1] ; \quad \tau=\frac{T}{2}(1+s), \quad s \in[-1, y],
$$




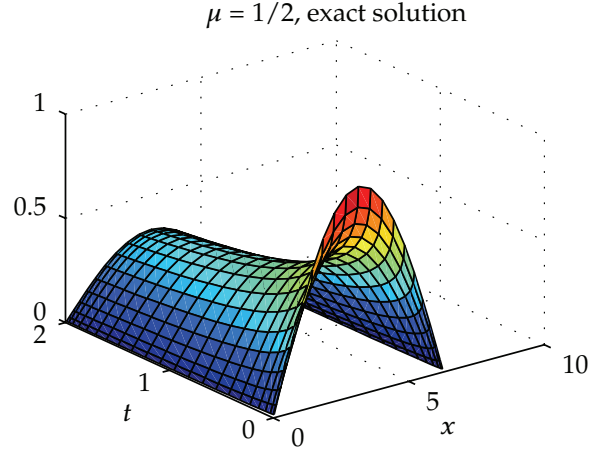

(a)

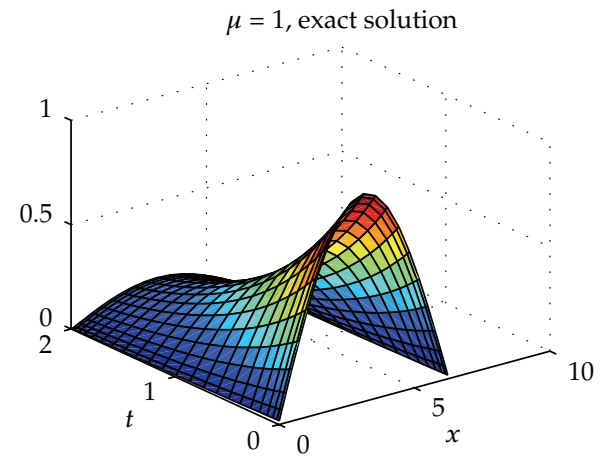

(c)

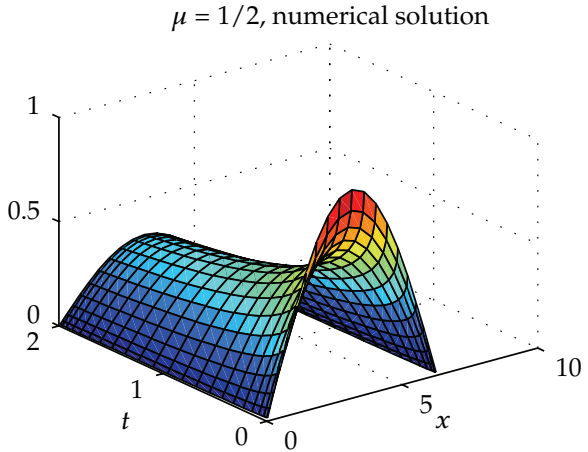

(b)

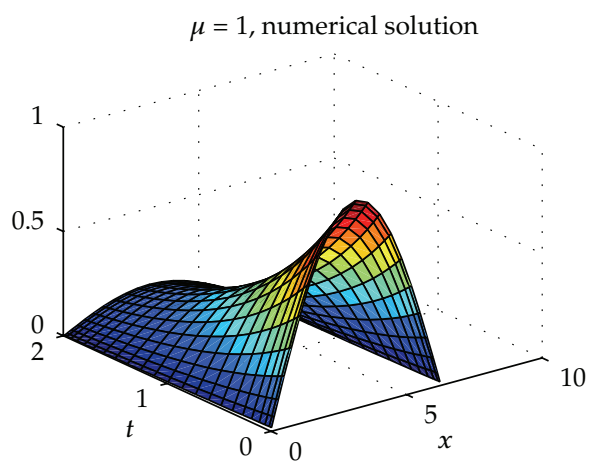

(d)

Figure 10: Exact and numerical solutions.

then the singular problems (3.5) can be rewritten as

$$
v(x, y)=g(x, y)+\frac{(T(1+y) / 2)^{1-\mu}}{\Gamma(\mu)}\left(\frac{T}{2}\right)^{2 \mu-1} \int_{-1}^{y} \frac{\mathcal{L}(x, s)}{(y-s)^{1-\mu}(1+s)^{1-\mu}} d s,
$$

where $y \in[-1,1]$, and

$$
v(x, y)=\bar{u}\left(x, \frac{T}{2}(1+y)\right), \quad g(x, y)=\bar{f}\left(x, \frac{T}{2}(1+y)\right) .
$$

For the collocation methods, (3.8) holds at the Gauss-Lobatto collocation points $\left\{x_{i}\right\}_{i=0}^{N}$ and Jacobi collocation points $\left\{y_{j}\right\}_{j=0}^{N}$ with Jacobi weight functions $\omega(y)=\left(1-y^{2}\right)^{\mu-1}$ on $[-1,1]$, namely,

$$
v\left(x_{i}, y_{j}\right)=g\left(x_{i}, y_{j}\right)+\frac{\left(T\left(1+y_{j}\right) / 2\right)^{1-\mu}}{\Gamma(\mu)}\left(\frac{T}{2}\right)^{2 \mu-1} \int_{-1}^{y_{j}} \frac{\mathcal{L v}\left(x_{i}, s\right) d s}{\left(y_{j}-s\right)^{1-\mu}(1+s)^{1-\mu}}
$$




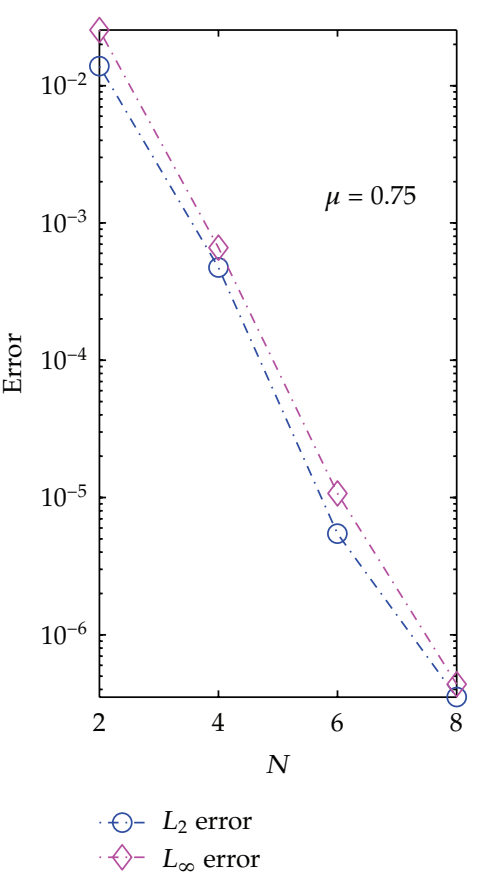

(a)



(b)

Figure 11: $y$-axis is $\log$ scale. (a) Error varying with the polynomial degree $N$. (b) Error varying with the polynomial degree $M$.

By using the following variable change:

$$
s=s_{j}(\theta)=\frac{1+y_{j}}{2} \theta+\frac{y_{j}-1}{2}, \quad \theta \in[-1,1]
$$

we can rewrite (3.10) as follows:

$$
\begin{aligned}
& v\left(x_{i}, y_{j}\right)=g\left(x_{i}, y_{j}\right)+\frac{\left[T\left(1+y_{j}\right) / 2\right]^{1-\mu}}{\Gamma(\mu)}\left(\frac{T\left(1+y_{j}\right) / 2}{2}\right)^{2 \mu-1} \int_{-1}^{1} \frac{\mathcal{L v}_{v}\left(x_{i}, s_{j}(\theta)\right)}{\left(1-\theta^{2}\right)^{1-\mu}} d \theta \\
& =g\left(x_{i}, y_{j}\right)+\frac{\left[T\left(1+y_{j}\right) / 2\right]^{\mu}}{2^{2 \mu-1} \Gamma(\mu)}\left(\mathcal{L} v\left(x_{i}, s_{j}(\cdot)\right), 1\right)_{\omega} .
\end{aligned}
$$

We first use $v_{i}^{j}, 0 \leq i \leq N ; 0 \leq j \leq M$ to indicate the approximate value for $v\left(x_{i}, y_{j}\right)$, then we can use

$$
v_{N}^{M}(x, y)=\sum_{n=0}^{N} \sum_{m=0}^{M} v_{n}^{m} h_{n}(x) F_{m}(y)
$$


to approximate the function $v(x, y)$, where $h_{n}(x)$ is the $n$th Lagrange interpolation polynomial associated with the collocation points $\left\{x_{i}\right\}_{i=0}^{N}$ and $F_{m}(y)$ is the $m$ th Lagrange interpolation polynomial associated with the collocation points $\left\{y_{i}\right\}_{i=0}^{M}$.

Using a $(M+1)$-point Gauss quadrature formula relative to the Jacobi weights $\left\{\omega_{j}\right\}_{j=0}^{M}$, (3.12) can be approximated by

$$
\begin{aligned}
& v_{i}^{j}= g\left(x_{i}, y_{j}\right)+\frac{\left[T\left(1+y_{j}\right) / 2\right]^{\mu}}{2^{2 \mu-1} \Gamma(\mu)}\left(\rho v_{N}^{M}\left(x_{i}, s_{j}(\cdot)\right), 1\right)_{M} \\
&= g\left(x_{i}, y_{j}\right)+\frac{\left[T\left(1+y_{j}\right) / 2\right]^{\mu}}{2^{2 \mu-1} \Gamma(\mu)} \\
& \times \sum_{k=0}^{M}\left(\sum_{n=0}^{N} \sum_{m=0}^{M} v_{n}^{m}\left(-\lambda^{2} h_{n}\left(x_{i}\right)-v h_{n}^{\prime}\left(x_{i}\right)+D h_{n}^{\prime \prime}\left(x_{i}\right)\right) F_{m}\left(s_{j}\left(\theta_{k}\right)\right)\right) \omega_{k}, \\
& \forall i=1, \ldots, N-1 ; j=0,1, \ldots, M,
\end{aligned}
$$

where the set $\left\{\theta_{k}\right\}_{k=0}^{M}$ coincides with the collocation points $\left\{y_{j}\right\}_{j=0}^{M}$ on $[-1,1]$.

Then the collocation spectral method is to seek $v_{N}^{M}(x, y)$ of the form (3.13) such that $v_{i}^{j}$ satisfies the above collocation equations (3.14) for $1 \leq i \leq N-1,0 \leq j \leq M$.

\section{Numerical Results with a Collocation Spectral Approximation}

In order to demonstrate the effectiveness of the proposed time-space collocation spectral method, some examples are now presented with Dirichlet boundary conditions, Neumann boundary conditions, and mixed boundary conditions.

For completeness sake, the implementation is briefly described here. To simplify the computation, we rewrite the above collocation equations (3.14) into the following:

$$
\begin{array}{r}
v_{i}^{j}=g_{i}^{j}+\sum_{n=0}^{N} \sum_{m=0}^{M} v_{n}^{m}\left(-\lambda^{2} h_{n}\left(x_{i}\right)-v h_{n}^{\prime}\left(x_{i}\right)+D h_{n}^{\prime \prime}\left(x_{i}\right)\right)\left(b_{j} \sum_{k=0}^{M} F_{m}\left(s_{j}\left(\theta_{k}\right)\right) \omega_{k}\right) \\
=g_{i}^{j}+\sum_{n=0}^{N} \sum_{m=0}^{M} d_{i n} a_{j m} v_{n}^{m}=g_{i}^{j}+\sum_{n=1}^{N-1} \sum_{m=0}^{M} d_{i n} a_{j m} v_{n}^{m}+\sum_{m=0}^{M}\left(d_{i 0} v_{0}^{m}+d_{i N} v_{N}^{m}\right) a_{j m} \\
\forall i=1, \ldots, N-1 ; j=0,1, \ldots, M,
\end{array}
$$

where

$$
\begin{gathered}
b_{j}=\frac{\left[T\left(1+y_{j}\right) / 2\right]^{\mu}}{2^{2 \mu-1} \Gamma(\mu)}, \quad a_{j m}=b_{j} \sum_{k=0}^{M} F_{m}\left(s_{j}\left(\theta_{k}\right)\right) \omega_{k}, \\
g_{i}^{j}=g\left(x_{i}, y_{j}\right), \quad d_{i n}=-\lambda^{2} h_{n}\left(x_{i}\right)-v h_{n}^{\prime}\left(x_{i}\right)+D h_{n}^{\prime \prime}\left(x_{i}\right) .
\end{gathered}
$$


In our numerical tests, we use the Chebyshev Gauss-Lobatto collocation points

$$
x_{i}=a+\frac{1}{2}\left(1-\cos \frac{i \pi}{N}\right)(b-a), \quad i=0,1, \ldots, N
$$

with the associated weights

$$
\bar{\omega}_{0}=\bar{\omega}_{N}=\frac{(b-a) \pi}{4 N}, \quad \bar{\omega}_{i}=\frac{(b-a) i \pi}{2 N}, \quad i=1, \ldots, N-1
$$

in the space. The other kinds Gauss-Lobatto collocation points (such as Legendre GaussLobatto collocation points) also can be used. The advantage of Gauss-Lobatto points is that they include the boundary points, which means we can apply boundary conditions there. For the time, the Jacobi Gauss collocation points are used for $\left\{y_{j}\right\}_{j=0}^{M}$ with the associated weights $\left\{\omega_{j}\right\}_{j=0}^{M}$ and other kinds of the Jacobi collocation points also suit to be used.

Let us set

$$
\begin{aligned}
& \mathbf{V}_{\mathbf{N}}^{\mathbf{M}}=\left[v_{1}^{0}, v_{2}^{0}, \ldots, v_{N-1}^{0}, v_{1}^{1}, \ldots, v_{N-1}^{1}, \ldots, v_{N-1}^{M}\right]^{T} \\
& \mathbf{G}_{\mathbf{N}}^{\mathbf{M}}=\left[g_{1}^{0}, g_{2}^{0}, \ldots, g_{N-1}^{0}, g_{1}^{1}, \ldots, g_{N-1}^{1}, \ldots, g_{N-1}^{M}\right]^{T} ;
\end{aligned}
$$

let $\mathbf{A}=\left(a_{i j}\right)$ be a matrix of $(M+1)$ by $(M+1)$, and $\mathbf{D}=\left(d_{i j}\right)$ is a matrix of $(N-1)$ by $(N-1)$.

\subsection{Implementation of Dirichlet Boundary Conditions}

The Dirichlet boundary conditions are directly applied in (4.1) and give numerical solutions on boundary in the following way:

$$
\begin{aligned}
& v_{0}^{m}=\left(\frac{T\left(1+y_{m}\right)}{2}\right)^{1-\mu}\left[\varphi_{1}\left(\frac{T\left(1+y_{m}\right)}{2}\right)-\phi(a)\right] \\
& v_{N}^{m}=\left(\frac{T\left(1+y_{m}\right)}{2}\right)^{1-\mu}\left[\varphi_{2}\left(\frac{T\left(1+y_{m}\right)}{2}\right)-\phi(b)\right] .
\end{aligned}
$$

We set

$$
\overline{\mathbf{G}}_{\mathbf{N}}^{\mathbf{M}}=\left[\bar{g}_{1}^{0}, \bar{g}_{2}^{0}, \ldots, \bar{g}_{N-1}^{0}, \bar{g}_{1}^{1}, \ldots, \bar{g}_{N-1}^{1}, \ldots, \bar{g}_{N-1}^{M}\right]^{T}
$$

where

$$
\bar{g}_{i}^{j}=\sum_{m=0}^{M}\left(d_{i 0} v_{0}^{m}+d_{i N} v_{N}^{m}\right) a_{j m} .
$$


Thus, the numerical scheme (4.1) leads to a system of equation of the form

$$
\mathbf{V}_{\mathbf{N}}^{\mathrm{M}}=\mathbf{F}_{\mathrm{N}}^{\mathrm{M}}+\mathrm{CV}_{\mathrm{N}}^{\mathrm{M}}
$$

where $\mathbf{F}_{\mathbf{N}}^{\mathbf{M}}=\mathbf{G}_{\mathbf{N}}^{\mathbf{M}}+\overline{\mathbf{G}}_{\mathbf{N}}^{\mathbf{M}}, \mathbf{C}=\left[a_{i j} \mathbf{D}\right]$ is a matrix of $(N-1) \times(M+1)$ by $(N-1) \times(M+1)$.

\subsection{Implementation of Neumann Boundary Conditions}

The Neumann boundary conditions (2.3) at $x=a$ and $x=b$ can be approximated as

$$
\begin{gathered}
\partial_{x} v_{N}^{M}\left(x_{0}, y_{m}\right)=\sum_{n=0}^{N} v_{n}^{m} h_{n}^{\prime}\left(x_{0}\right)=\left(\frac{T\left(1+y_{m}\right)}{2}\right)^{1-\mu}\left[\varphi_{1}\left(\frac{T\left(1+y_{m}\right)}{2}\right)-\phi^{\prime}\left(x_{0}\right)\right] \doteq r_{m}^{(1)} \\
\partial_{x} v_{N}^{M}\left(x_{N}, y_{m}\right)=\sum_{n=0}^{N} v_{n}^{m} h_{n}^{\prime}\left(x_{N}\right)=\left(\frac{T\left(1+y_{m}\right)}{2}\right)^{1-\mu}\left[\varphi_{2}\left(\frac{T\left(1+y_{m}\right)}{2}\right)-\phi^{\prime}\left(x_{N}\right)\right] \doteq r_{m}^{(2)} .
\end{gathered}
$$

Equation (4.10) can be written as follows:

$$
\begin{aligned}
& h_{0}^{\prime}\left(x_{0}\right) v_{0}^{m}+h_{N}^{\prime}\left(x_{0}\right) v_{N}^{m}=r_{m}^{(1)}-\sum_{n=1}^{N-1} v_{n}^{m} h_{n}^{\prime}\left(x_{n}\right) \\
& h_{0}^{\prime}\left(x_{N}\right) v_{0}^{m}+h_{N}^{\prime}\left(x_{N}\right) v_{N}^{m}=r_{m}^{(2)}-\sum_{n=1}^{N-1} v_{n}^{m} h_{n}^{\prime}\left(x_{n}\right)
\end{aligned}
$$

Solving (4.11) for $v_{0}^{m}$ and $v_{N}^{m}$, we get

$$
\begin{gathered}
v_{0}^{m}=\frac{h_{N}^{\prime}\left(x_{0}\right)\left(r_{m}^{(2)}-\sum_{n=1}^{N-1} v_{n}^{m} h_{n}^{\prime}\left(x_{N}\right)\right)-h_{N}^{\prime}\left(x_{N}\right)\left(r_{m}^{(1)}-\sum_{n=1}^{N-1} v_{n}^{m} h_{n}^{\prime}\left(x_{0}\right)\right)}{h_{N}^{\prime}\left(x_{0}\right) h_{0}^{\prime}\left(x_{N}\right)-h_{0}^{\prime}\left(x_{0}\right) h_{N}^{\prime}\left(x_{N}\right)}, \\
v_{N}^{m}=\frac{h_{0}^{\prime}\left(x_{N}\right)\left(r_{m}^{(1)}-\sum_{n=1}^{N-1} v_{n}^{m} h_{n}^{\prime}\left(x_{0}\right)\right)-h_{0}^{\prime}\left(x_{0}\right)\left(r_{m}^{(2)}-\sum_{n=1}^{N-1} v_{n}^{m} h_{n}^{\prime}\left(x_{N}\right)\right)}{h_{N}^{\prime}\left(x_{0}\right) h_{0}^{\prime}\left(x_{N}\right)-h_{0}^{\prime}\left(x_{0}\right) h_{N}^{\prime}\left(x_{N}\right)} .
\end{gathered}
$$


International Journal of Differential Equations

Then the last right term of (4.1) can be written as follows:

$$
\begin{aligned}
\sum_{m=0}^{M}\left(d_{i 0} v_{0}^{m}+d_{i N} v_{N}^{m}\right) a_{j m}= & \sum_{m=0}^{M}\left(c_{i}^{(1)} r_{m}^{(1)}-c_{i}^{(2)} r_{m}^{(2)}\right) a_{j m} \\
& +\sum_{m=0}^{M}\left(c_{i}^{(2)} \sum_{n=1}^{N-1} v_{n}^{m} h_{n}^{\prime}\left(x_{N}\right)-c_{i}^{(1)} \sum_{n=1}^{N-1} v_{n}^{m} h_{n}^{\prime}\left(x_{0}\right)\right) a_{j m} \\
= & \sum_{m=0}^{M}\left(c_{i}^{(1)} r_{m}^{(1)}-c_{i}^{(2)} r_{m}^{(2)}\right) a_{j m} \\
& +\sum_{m=0}^{M} \sum_{n=1}^{N-1} v_{n}^{m}\left(c_{i}^{(2)} h_{n}^{\prime}\left(x_{N}\right)-c_{i}^{(1)} h_{n}^{\prime}\left(x_{0}\right)\right) a_{j m}
\end{aligned}
$$

where

$$
c_{i}^{(1)}=\frac{h_{0}^{\prime}\left(x_{N}\right) d_{i N}-h_{N}^{\prime}\left(x_{N}\right) d_{i 0}}{h_{N}^{\prime}\left(x_{0}\right) h_{0}^{\prime}\left(x_{N}\right)-h_{0}^{\prime}\left(x_{0}\right) h_{N}^{\prime}\left(x_{N}\right)}, \quad c_{i}^{(2)}=\frac{h_{0}^{\prime}\left(x_{0}\right) d_{i N}-h_{N}^{\prime}\left(x_{0}\right) d_{i 0}}{h_{N}^{\prime}\left(x_{0}\right) h_{0}^{\prime}\left(x_{N}\right)-h_{0}^{\prime}\left(x_{0}\right) h_{N}^{\prime}\left(x_{N}\right)} .
$$

Let

$$
\widetilde{\mathbf{G}}_{\mathbf{N}}^{\mathrm{M}}=\left[\tilde{g}_{1}^{0}, \widetilde{g}_{2}^{0}, \ldots, \tilde{g}_{N-1}^{0}, \tilde{g}_{1}^{1}, \ldots, \tilde{g}_{N-1}^{1}, \ldots, \widetilde{g}_{N-1}^{M}\right]^{T},
$$

where

$$
\begin{gathered}
\tilde{g}_{i}^{j}=\sum_{m=0}^{M}\left(c_{i}^{(1)} r_{m}^{(1)}-c_{i}^{(2)} r_{m}^{(2)}\right) a_{j m}, \\
\tilde{d}_{i n}=d_{i n}+c_{i}^{(2)} h_{n}^{\prime}\left(x_{N}\right)-c_{i}^{(1)} h_{n}^{\prime}\left(x_{0}\right) \\
=-\lambda^{2} h_{n}\left(x_{i}\right)-v h_{n}^{\prime}\left(x_{i}\right)+D h_{n}^{\prime \prime}\left(x_{i}\right)+c_{i}^{(2)} h_{n}^{\prime}\left(x_{N}\right)-c_{i}^{(1)} h_{n}^{\prime}\left(x_{0}\right), \\
\tilde{\mathbf{D}}=\left(\tilde{d}_{i j}\right) \text { is a matrix of }(N-1) \text { by }(N-1) .
\end{gathered}
$$

Then the numerical scheme (4.1) leads to a system of equation of the form

$$
\mathbf{V}_{\mathrm{N}}^{\mathrm{M}}=\mathrm{F}_{\mathrm{N}}^{\mathrm{M}}+\mathrm{CV}_{\mathrm{N}}^{\mathrm{M}}
$$

where $\mathbf{F}_{\mathbf{N}}^{\mathbf{M}}=\mathbf{G}_{\mathbf{N}}^{\mathbf{M}}+\tilde{\mathbf{G}}_{\mathbf{N}}^{\mathbf{M}}, \mathbf{C}=\left[a_{i j} \tilde{\mathbf{D}}\right]$ is a matrix of $(N-1) \times(M+1)$ by $(N-1) \times(M+1)$.

Remark 4.1. The implementation for the mixed boundary conditions also can be derived by (4.6) and (4.12). 


\subsection{Numerical Experiments}

In this subsection, the proposed numerical scheme is applied to several test problems to show the efficiency and spectral accuracy. formulas:

In each example, we have calculated $L_{2}$ errors and $L_{\infty}$ errors given by the following

$$
\begin{gathered}
L_{2} \text { error }=\sqrt{\sum_{i=0}^{N} \sum_{j=0}^{M}\left[u_{i}^{j}-u\left(x_{i}, t_{j}\right)\right]^{2} w_{i} \bar{w}_{j}} ; \\
L_{\infty} \text { error }=\max _{i, j}\left|u_{i}^{j}-u\left(x_{i}, t_{j}\right)\right|,
\end{gathered}
$$

where $u_{i}^{j}$ is the numerical approximation solutions of the exact solutions $u\left(x_{i}, t_{j}\right)$.

Example 4.2 (Dirichlet Boundary Conditions). In this example, we consider the following time fractional diffusion equations:

$$
\begin{gathered}
D_{t}^{\mu} u(x, t)=\frac{\partial^{2} u(x, t)}{\partial x^{2}}+f(x, t), \quad x \in(-1,1), t \in(0, T) \\
u(x, 0)=0, \quad x \in(a, b) \\
u(-1, t)=u(1, t)=0, \quad t \in(0, T)
\end{gathered}
$$

where

$$
f(x, t)=\frac{\Gamma(1+\beta)}{\Gamma(1+\beta-\mu)} t^{\beta-\mu} \sin (2 \pi x)+4 \pi^{2} t^{\beta} \sin (2 \pi x) .
$$

The exact solution is given by

$$
u(x, t)=t^{\beta} \sin (2 \pi x), \quad \beta>-1 .
$$

In Figure 1, we plot the exact and numerical solutions, and, in Figure 2, we represent the associated errors field. Here we set $N=M=22, \mu=0.8$, and $\beta=-0.2$. The results in Figures 1 and 2 denote that the numerical solution using the proposed collocation spectral method is excellent in agreement with the exact solution at the whole domain. The efficiency of this collocation spectral method can be further confirmed by Figures 3-4, which are the comparison of the exact solution and numerical solution when the space variable $x$ or time variable $t$ is fixed for various $\mu$ and $\beta$; here $N=M=22$.

The main purpose of the numerical test is to check the convergence behavior of numerical solutions with respect to the polynomial degrees $M$ and $N$ for several $\mu$, especially the convergence in time because of the fractional derivative in time. In order to investigate the spatial accuracy when $N$ increases, we take $M$ larger enough so that the time discretization errors are negligible compared with the spatial discretization errors. Similary, for the temporal accuracy, we must keep $N$ large enough to preclude spatial errors. $L_{2}$ errors 
Table 1: $L_{2}$ errors and $L_{\infty}$ errors varying with $(N, M)$ and $\mu$.

\begin{tabular}{lcccccc}
\hline \multirow{2}{*}{$(N, M)$} & \multicolumn{2}{c}{$\mu=0.1$} & \multicolumn{2}{c}{$\mu=0.6$} & \multicolumn{2}{c}{$\mu=0.99$} \\
& $L_{2}$ error & $L_{\infty}$ error & $L_{2}$ error & $L_{\infty}$ error & $L_{2}$ error & $L_{\infty}$ error \\
\hline$(6,22)$ & $8.3822 e-004$ & $2.7393 e-004$ & $1.8302 e-004$ & $1.7793 e-004$ & $8.4539 e-005$ & $1.2560 e-004$ \\
$(8,22)$ & $8.8873 e-006$ & $2.3883 e-006$ & $1.6207 e-006$ & $1.5843 e-006$ & $7.6277 e-007$ & $1.1439 e-006$ \\
$(10,22)$ & $5.1229 e-006$ & $1.8179 e-006$ & $4.4081 e-008$ & $1.2032 e-007$ & $4.3509 e-009$ & $6.6034 e-009$ \\
$(22,6)$ & 0.0013 & $3.8771 e-004$ & $5.0251 e-005$ & $5.6230 e-005$ & $2.3470 e-007$ & $2.9261 e-007$ \\
$(22,8)$ & $3.7516 e-004$ & $1.1631 e-004$ & $1.0916 e-005$ & $1.4832 e-005$ & $4.5707 e-008$ & $6.9221 e-008$ \\
$(22,10)$ & $1.4591 e-004$ & $4.6309 e-005$ & $3.2976 e-006$ & $5.2297 e-006$ & $1.2453 e-008$ & $2.1838 e-008$ \\
\hline
\end{tabular}

and $L_{\infty}$ errors in semilog scale varying with the polynomial degree $N$ (see (a)) or $M$ (see (b)) are shown in Figures 5 and 6 for $\mu=0.05,0.75$ with $\beta=2.2$, and Figure 7 for $\mu=1$ with $\beta=1.2$. It is clear that the spectral convergence is achieved both of spatial and temporal errors. This indicates that the convergence in space and time of the time-space collocation spectral method is exponential, even though for the classical diffusion equation $(\mu=1)$.

Example 4.3 (Neumann Boundary Conditions). Consider the time fractional advectiondiffusion equation with Neumann boundary conditions

$$
\begin{gathered}
D_{t}^{\mu} u(x, t)=-v \frac{\partial u(x, t)}{\partial x}+D \frac{\partial^{2} u(x, t)}{\partial x^{2}}+f(x, t), \quad x \in(3,5), t>0, \\
u(x, 0)=0, \quad x \in(3,5), \\
\partial_{x} u(3, t)=\frac{\pi t^{2}}{2}, \quad \partial_{x} u(5, t)=\frac{-\pi t^{2}}{2}, \quad t>0 .
\end{gathered}
$$

We choose the suitable source term $f(x, t)$ to obtain the exact solution

$$
u(x, t)=t^{2} \cos \left(\frac{\pi x}{2}\right) .
$$

In Table $1, L_{2}$ errors and $L_{\infty}$ errors varying with $(N, M)$ and $\mu$ are given, and it indicates the efficiency of the present technique.

In Figure 8, we plot $L_{2}$ errors and $L_{\infty}$ errors in semilog scale. Figures $8(\mathrm{a})$ and $8(\mathrm{~b})$ are concerned with the spatial errors with $M=16$ and the temporal errors with $N=16$, respectively. As expected, the spatial and temporal spectral convergence is achieved. Our greatest interest is to check the convergence in time because of the fractional derivative in time. So we plot the $L_{2}$ errors and $L_{\infty}$ errors as functions of $M$ for several more values $\mu$ in Figure 9, where $N=16$. This graph shows that the spectral convergence in time can be reached, and that the time-space collocation spectral method also works well for the classical advection-diffusion equation. 
Example 4.4 (Mixed Boundary Conditions). Consider the time fractional reactionsubdiffusion equation

$$
\begin{gathered}
D_{t}^{\mu} u(x, t)=-\frac{3}{4} u(x, t)+\frac{\partial^{2} u(x, t)}{\partial x^{2}}, \quad x \in(0,2 \pi), t>0, \\
u(x, 0)=\sin \left(\frac{x}{2}\right), \quad x \in(0,2 \pi) \\
u(0, t)=0, \quad \partial_{x} u(2 \pi, t)=-\frac{1}{2} E_{\mu}\left(-t^{\mu}\right) .
\end{gathered}
$$

The exact solution is given by

$$
u(x, t)=E_{\mu}\left(-t^{\mu}\right) \sin \left(\frac{x}{2}\right)
$$

In Figure 10, we plot the visual fields of the exact and numerical solutions. Comparing Figures 10(a) and 10(c), and Figures 10(b) and 10(d), we see that the numerical solution is in good agreement with the exact solution; the shapes on the space variable are similar to Sine function. In addition, we can see that the solutions decay over time from the initial state. Comparing Figures 10(a) and 10(b) with Figures 10(c) and 10(d), (a) and (b) decay faster in the beginning and the trend is the opposite of (c) and (d). This is consistent with the behavior of the function $E_{\alpha}(z)$. For $0<t<0.77, E_{1 / 2}\left(-t^{1 / 2}\right)$ decays faster than $E_{1}(-t)$, whereas for $t>0.77$, the trend is just the opposite.

We plot in Figure 11 the $L_{2}$ errors and $L_{\infty}$ errors versus $N$ with $M=16$ in 11 (a) and versus $M$ with $N=16$ in 11(b). As can be seen in Figure 11, the proposed method provides spatial and temporal spectral convergence for $L_{2}$ errors and $L_{\infty}$ errors.

\section{Conclusion}

This paper proposes a time-space collocation spectral method for a class of time fractional differential equations with Caputo derivatives. The proposed method also works well for the corresponding classical integer-order partial differential equations $(\mu=1)$, and it differs from (and is simpler than) the existing time-space spectral methods which are based on the Petrov-Galerkin or Dual-Petrov-Galerkin formulation. The main advantage of the present scheme is that it gives very accurate convergency by choosing less number of grid points and the problem can be solved up to big time, and the storage requirement due to the time memory effect can be considerably reduced. At the same time, the technique is also simpler and easier to apply to multidimensional problems than the existing Galerkin spectral method such as the methods in $[19,20]$.

\section{References}

[1] I. Podlubny, Fractional Differential Equations, vol. 198 of Mathematics in Science and Engineering, Academic Press, San Diego, Calif, USA, 1999.

[2] W. Wyss, "The fractional diffusion equation," Journal of Mathematical Physics, vol. 27, no. 11, pp. 27822785, 1986. 
[3] W. R. Schneider and W. Wyss, "Fractional diffusion and wave equations," Journal of Mathematical Physics, vol. 30, no. 1, pp. 134-144, 1989.

[4] F. Huang and F. Liu, "The time fractional diffusion equation and the advection-dispersion equation," The Australian \& New Zealand Industrial and Applied Mathematics Journal, vol. 46, no. 3, pp. 317-330, 2005.

[5] F. Huang and B. Guo, "General solutions to a class of time fractional partial differential equations," Applied Mathematics and Mechanics, vol. 31, no. 7, pp. 815-826, 2010.

[6] Y. Luchko and R. Gorenoo, "The initial value problem for some fractional differen-tial equations with the Caputo derivatives," Preprint Series A08-98, Fachbreich Mathematik und Informatik, Freic Universitat Berlin, 1998.

[7] N. T. Shawagfeh, "Analytical approximate solutions for nonlinear fractional differential equations," Applied Mathematics and Computation, vol. 131, no. 2-3, pp. 517-529, 2002.

[8] S. G. Samko, A. A. Kilbas, and O. I. Marichev, Fractional Integrals and Derivatives, Gordon and Breach Science Publishers, Yverdon, Switzerland, 1993.

[9] J. Chen, F. Liu, and V. Anh, "Analytical solution for the time-fractional telegraph equation by the method of separating variables," Journal of Mathematical Analysis and Applications, vol. 338, no. 2, pp. 1364-1377, 2008.

[10] M. M. Meerschaert and C. Tadjeran, "Finite difference approximations for fractional advectiondispersion flow equations," Journal of Computational and Applied Mathematics, vol. 118, pp. 283-299, 2004.

[11] T. A. M. Langlands and B. I. Henry, "The accuracy and stability of an implicit solution method for the fractional diffusion equation," Journal of Computational Physics, vol. 205, no. 2, pp. 719-736, 2005.

[12] Y. Lin and C. Xu, "Finite difference/spectral approximations for the time-fractional diffusion equation," Journal of Computational Physics, vol. 225, no. 2, pp. 1533-1552, 2007.

[13] C.-M. Chen, F. Liu, and K. Burrage, "Finite difference methods and a Fourier analysis for the fractional reaction-subdiffusion equation," Applied Mathematics and Computation, vol. 198, no. 2, pp. 754-769, 2008.

[14] I. Podlubny, A. Chechkin, T. Skovranek, Y. Chen, and B. M. Vinagre Jara, "Matrix approach to discrete fractional calculus. II. Partial fractional differential equations," Journal of Computational Physics, vol. 228, no. 8, pp. 3137-3153, 2009.

[15] G. J. Fix and J. P. Roop, "Least squares finite-element solution of a fractional order two-point boundary value problem," Computers \& Mathematics with Applications, vol. 48, no. 7-8, pp. 1017-1033, 2004.

[16] J. P. Roop, "Computational aspects of FEM approximation of fractional advection dispersion equations on bounded domains in $R^{2}$, , Journal of Computational and Applied Mathematics, vol. 193, no. 1, pp. 243-268, 2006.

[17] W. H. Deng, "Finite element method for the space and time fractional Fokker-Planck equation," SIAM Journal on Numerical Analysis, vol. 47, no. 1, pp. 204-226, 2008/09.

[18] E. Hanert, "A comparison of three Eulerian numerical methods for fractional-order transport models," Environmental Fluid Mechanics, vol. 10, no. 1, pp. 7-20, 2010.

[19] E. Hanert, "On the numerical solution of space-time fractional diffusion models," Computers and Fluids, vol. 46, no. 1, pp. 33-39, 2011.

[20] X. Li and C. Xu, "A space-time spectral method for the time fractional diffusion equation," SIAM Journal on Numerical Analysis, vol. 47, no. 3, pp. 2108-2131, 2009.

[21] Y. Chen and T. Tang, "Convergence analysis of the Jacobi spectral-collocation methods for Volterra integral equations with a weakly singular kernel," Mathematics of Computation, vol. 79, no. 269, pp. 147-167, 2010. 


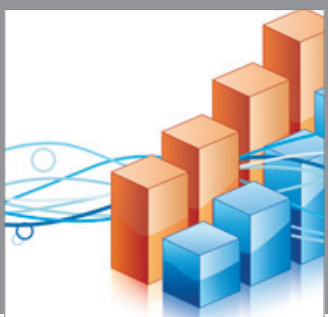

Advances in

Operations Research

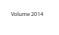

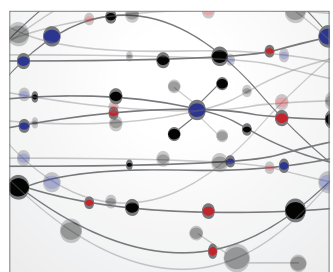

\section{The Scientific} World Journal
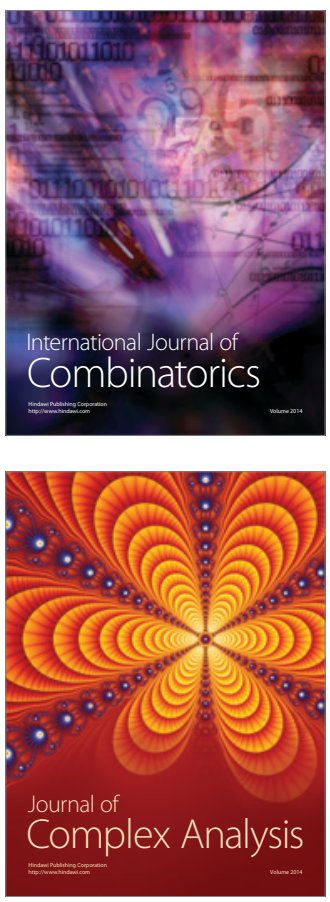

International Journal of

Mathematics and

Mathematical

Sciences
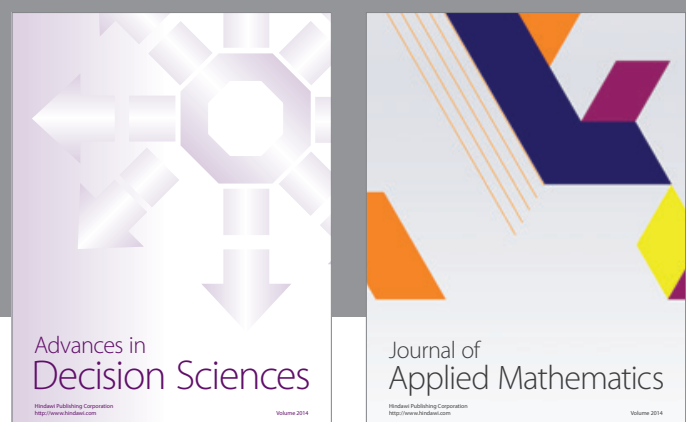

Journal of

Applied Mathematics
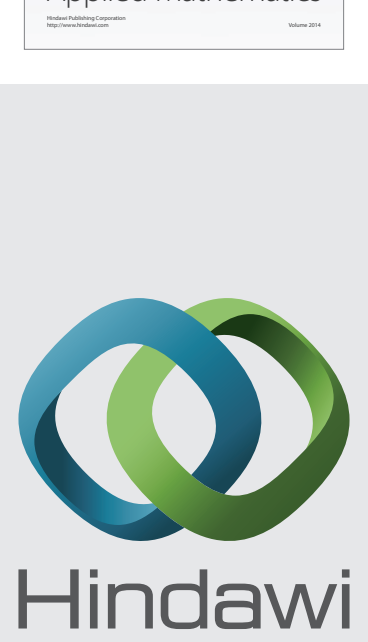

Submit your manuscripts at http://www.hindawi.com
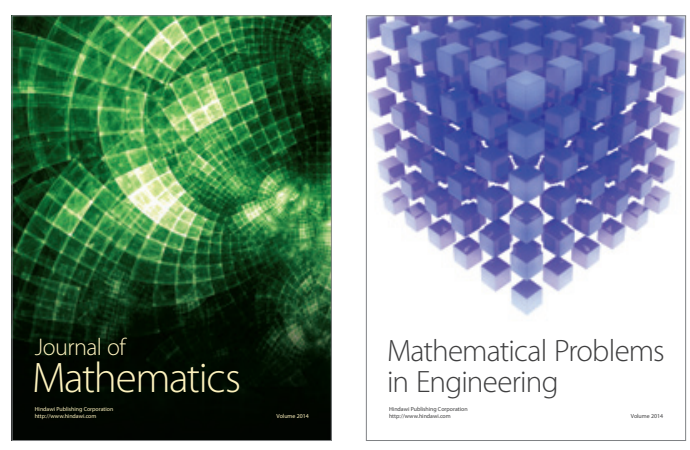

Mathematical Problems in Engineering
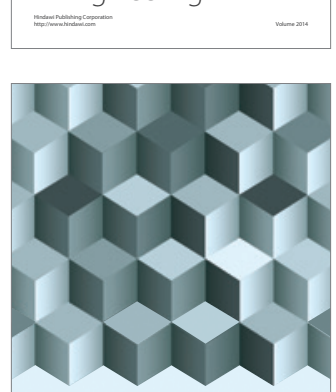

Journal of

Function Spaces
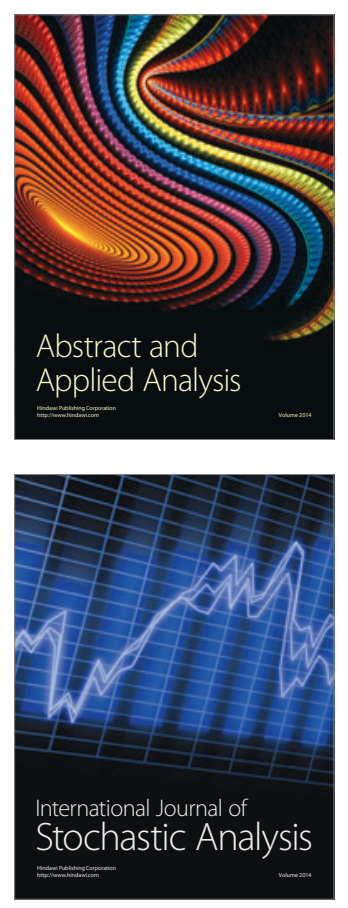



ournal of

Probability and Statistics

Promensencen
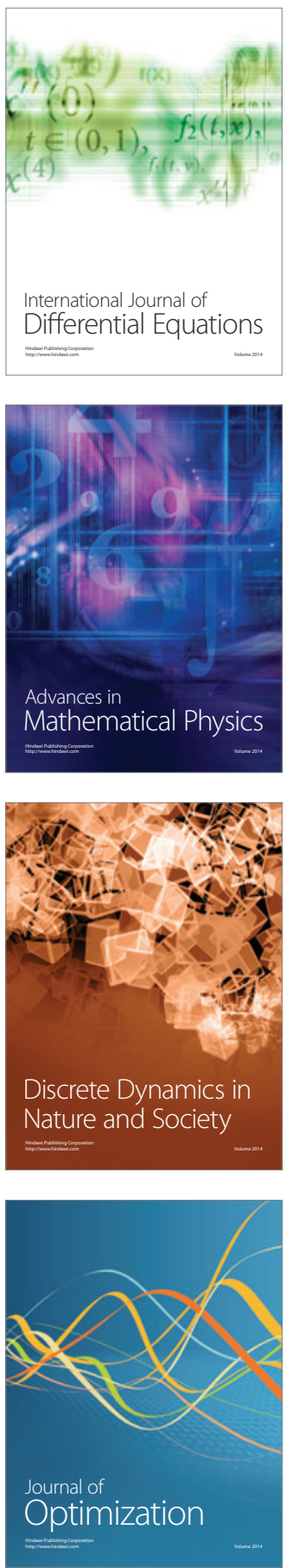